REPORTE DE TESIS DE POSGRADO

\title{
Aplicación gamificada para apoyo en la educación de personas con diabetes
}

\author{
Andrea Stephanía Arce-Jiménez, Norma Barón- \\ Ramírez, Juan José Contreras-Castillo
}

Publicado: 31 Octubre 2018

\begin{abstract}
Resumen
Este artículo presenta la propuesta del desarrollo de una aplicación móvil gamificada basada en el programa DiabetIMSS, que pretende apoyar a los pacientes con diabetes en su aprendizaje sobre su enfermedad y el cuidado de su salud.
\end{abstract}

Palabras clave: Aplicación; diabetes; educación para la salud; gamificación.

\section{Introducción}

La diabetes es una afección crónica que se desencadena cuando el organismo pierde su capacidad para producir suficiente insulina. Se presenta cuando el organismo no utiliza eficazmente la insulina que produce el páncreas o la producción es insuficiente.

La diabetes se clasifica en 4 categorías: diabetes mellitus tipo 1(insulinodependiente), diabetes mellitus tipo 2 (no insulinodependiente), diabetes mellitus gestacional y casos especiales generados por causas distintas como trasplantes, neonatal o enfermedades [1].

El Observatorio Mexicano de Enfermedades No Transmisibles (OMENT) calcula que en México hay un total de 1, 021,912 personas en tratamiento que viven con diabetes mellitus tipo 2 donde el 71.5 por ciento es representado por mujeres entre los 55 y 59 años [2].

El incremento de la enfermedad se debe al sedentarismo, enfermedades como la obesidad y sobrepeso, los antecedentes familiares pero también a estilos de vida poco saludables entre niños, adolescentes y adultos [3], [4].

El estilo de vida que tiene el paciente juega un papel fundamental antes y después de la detección de la enfermedad. Hábitos como el consumo de alcohol, la falta de actividad física, el consumo de tabaco y el tipo de alimentación son uno de los varios factores que se deben modificar si lo que se quiere es prevenir o mejorar la calidad de vida de los pacientes[4].

Los hábitos son comportamientos que implican actividades psicomotoras y cognitivas que se realizan generalmente de la misma forma sin que exista un control plenamente consciente y voluntario. Permiten satisfacer necesidades fisiológicas, afectivas y sociales [5].

Arce-Jiménez, AS., Barón-Ramírez, N., Contreras-Castillo, JJ.

Universidad de Colima

Colima, México

Email: andrea_arce@ucol.mx,norma@ucol.mx, juancont@ucol.mx
Un hábito se consolida en tres etapas: señal (reacción del cerebro por realizar una actividad), rutina (física, mental o emocional) y la recompensa (valoración de si vale la pena continuar) [6]. Para cambiar un hábito es necesario identificar la señal y de esta manera cambiar la rutina. Un concepto que permite, a través de la obtención de información formar estilos de vida saludables y modificar hábitos, es la educación para la salud.

La educación para la salud es el esfuerzo de proporcionar información y conocimientos relativos al mantenimiento y promoción de la salud, implica aspectos formales e informales [7]. Los aspectos formales podemos entenderlos como actividades dirigidas y programadas, los informales a los conocimientos que se obtienen de forma empírica.

Los 3 encargados de programas educativos para la atención de la diabetes en el gobierno del Estado de Colima y las instituciones de salud que se entrevistaron para este trabajo coinciden en que la diabetes es una enfermedad que solo se hereda cuando los hábitos que se promueven en casa son malos, por lo cual, es necesario educar a la población para modificarlos y que estos no se sigan transmitiendo de generación en generación y en consecuencia se presenten más casos de la enfermedad.

El diseño y desarrollo de la aplicación se basa en el programa DiabetIMSS del Instituto Mexicano del Seguro Social (IMSS) que realiza cursos mensuales presenciales para que sus pacientes aprendan a controlar y vivir con la enfermedad.

Haciendo uso de las tecnologías de información adaptaremos el programa DiabetIMSS a una aplicación móvil en la cual, los pacientes con diabetes podrán tener acceso a la información del programa. Con el fin de lograr el objetivo de educar a los pacientes de manera entretenida, se empleará la gamificación.

La gamificación es el proceso de jugar-pensando en conjunto con las mecánicas de juego para comprometer a los usuarios y que estos resuelvan problemas [8]. En otras palabras, la gamificación utiliza los elementos presentes en los videojuegos (ej. puntos y medallas) para generar en el usuario poco a poco un compromiso con las actividades que realiza.

Gamificando la aplicación se motiva a los usuarios a que cumplan con las tareas que mejoran su salud y a su vez con el uso frecuente identificarán las señales que les provocan cierto hábito y de esta manera cambiar su rutina, lo que a un mayor plazo terminará siendo una actividad en su vida cotidiana.

A través de entrevistas y conociendo los diferentes programas ofrecidos por instituciones de salud se diseñó la propuesta de la aplicación que se describe en el presente artículo. 


\section{Trabajos Relacionados}

Antes de comenzar a hablar del proyecto es necesario conocer la diferencia entre la gamificación y los juegos serios debido a que son conceptos que suelen confundirse. Los juegos serios son juegos que involucran al usuario y al mismo tiempo contribuyen a la consecución de un objetivo determinado más allá del entretenimiento ya sea que el usuario este consciente de ello o no [9].

La gamificación por su parte, hace uso de elementos de videojuegos) para contextos de no-juego con el propósito de motivar a las personas a completar sus tareas e incrementar su compromiso con las actividades que realiza [10].

Dadas estas definiciones se encuentra que la diferencia radica en que la gamificación es un conjunto de tareas y procedimientos ya establecidos que el usuario va realizando mientras que los juegos serios, el usuario va explorando las distintas formas de aprender y se dirige por un camino en el que el jugador va descubriendo lo que quiere aprender conforme el juego avanza.

La gamificación se ha utilizado principalmente para fines educativos y de marketing. Empresas como Nike utilizan aplicaciones gamificadas como Nike+ para promover la actividad física, pero van de la mano de sus productos con esto se logra fidelizar a la población con su marca [11].

En educación, esta Duolingo, una plataforma que permite a sus usuarios aprender distintos idiomas. Utiliza medallas y puntos como recompensas por cada lección terminada y genera alertas en el caso de que los usuarios no entren con la frecuencia esperada a la plataforma [12].

Respecto a la gamificación en la salud diversos investigadores han desarrollado aplicaciones para mejorar los estilos de vida de las personas, la mayor parte de ellas están destinadas a reducir la obesidad y sobrepeso. A continuación, se enlistan algunas de ellas:

\subsection{Food Quiz}

Es una aplicación para celulares cuyo principal objetivo es que los jóvenes aprendan a controlar el consumo de calorías. Consiste en mostrar preguntas sobre estimación de calorías en un alimento a partir de una imagen con opciones y otro ejercicio es el cálculo de las calorías, la aplicación les proporciona las fórmulas matemáticas que deben utilizar. En caso de que contesten de manera incorrecta se les proporciona información como retroalimentación y si la respuesta fue correcta se les otorgan puntos [13]. Los puntos sirven para establecer un listado de los participantes y que ellos conozcan en qué lugar se encuentran respecto a otros participantes.

\subsection{SHESHOP}

Es una aplicación que cuenta los pasos que realiza el usuario durante el día. La aplicación se diseñó con el fin de prevenir enfermedades mediante la activación física. En la aplicación se presentan distintos niveles y distintos tipos de ejercicios dependiendo si es un niño, joven o adulto quien la utiliza. Cuando el usuario completa un nivel recibe una nueva meta, la cual va incrementando conforme se pase de nivel [14].

\subsection{San-Poki}

Aplicación creada con la finalidad de que las personas adultas adquieran el hábito del ejercicio. Invita a sus usuarios a visitar lugares específicos caminando al estilo de "rally". Al llegar al lugar pueden toman una foto y por cada foto que hagan obtienen una medalla. Este juego genera competencia al mostrar a otros los logros que el usuario va realizando [15].
Las aplicaciones mencionadas se enfocan solamente en establecer hábitos que mejoren la calidad de vida de los pacientes con algún padecimiento y no educarlos de manera integral.

Lo que se pretende con la aplicación propuesta en este artículo, es que los pacientes aprendan y al mismo tiempo transiten hacía hábitos saludables, aprendiendo sobre su enfermedad, los mitos y realidades, temas de alimentación y actividad física, entre otros.

Gamificando cada una de las unidades se pretende que el usuario se motive y utilice la aplicación de manera constante sin perder el enfoque pedagógico

\section{Objetivo de la Investigación}

El objetivo es educar a pacientes con diabetes sobre las características de enfermedad y que aprendan a autocontrol sobre ella así como la mejora de hábitos. Por medio de una aplicación que les ofrezca llevar el historial de sus medidas (talla, peso, azúcar, presión), dar de altas recordatorios, establecer metas de pasos diarios (motivándolos a realizar actividad física) y lo más importante, un módulo donde encontrarán actividades educativas sobre temas importantes para la salud (nutrición, actividad física, cuidado personal, entre otros) que le permitan aprender sobre su enfermedad.

\section{Metodología}

Se visitaron 2 instituciones de salud pública del Estado de Colima, el Instituto de Seguridad y Servicios Sociales de los Trabajadores del Estado (ISSSTE) e IMSS, así como a la Secretaría de Salud del Gobierno del Estado con el propósito de conocer los programas educativos que ofrecen a los pacientes con diabetes. Se entrevistaron a los responsables de cada uno de los programas (uno por cada programa) y se obtuvo la siguiente información:

\subsection{DiabetIMSS}

Es un programa ofrecido por el (IMSS) cuya meta es el que el paciente lleve un adecuado control de la enfermedad. Se realizan doce sesiones durante un año dirigidas por especialistas. Al iniciar cada sesión se realiza una consulta médica rápida. Se explican temas sobre diabetes (definición), alimentación, actividad física, salud sexual y bucal, cuidado de los pies, prevención de caídas, entre otros.

Esta información fue obtenida al visitar la clínica familiar no. 19 ubicada en el municipio de Colima, donde el encargado de dicho programa en la clínica resalta el éxito de este ya que sus pacientes han disminuido sus niveles de hemoglobina glucosilada (valor de referencia) de manera significativa al final de las 12 sesiones.

\subsection{MIDE}

El Módulo Integral de Diabetes por Etapas (MIDE) es el programa a cargo del ISSSTE que se desarrolla en cuatro etapas: 1) Educativo, 2) Dieta, 3) Ejercicio, 4) Medicamento. El programa se dividió en etapas para que el paciente recordara los pasos más importantes para llevar un adecuado estilo de vida y el control de su enfermedad. La educación en el programa corre a cargo del doctor del paciente quien explica desde cómo se generó su enfermedad hasta que acciones debe seguir para no sufrir complicaciones.

El seguimiento a los pacientes se lleva a cabo cada tres meses durante un periodo de nueve meses a un año y se lleva un registro de los niveles de hemoglobina glucosilada en el paciente para verificar la estabilidad de su nivel de salud. 


\subsection{Grupos de Ayuda Mutua.}

La Secretaría de Salud lleva a cabo los grupos de ayuda mutua para difundir y promover la adopción de hábitos de vida saludables. Estos grupos se realizan mensualmente y el paciente tiene la responsabilidad de asistir. Los principales temas que revisan son actividad física, alimentación, prevención y control de riesgos, entre otros.

El grupo es supervisado por una mesa directiva (personal especializado en el tema) que da el visto bueno al acta constitutiva que se crea para iniciar el grupo en la cual se registran los participantes y el plan de trabajo que se seguirá para lograr los objetivos de acreditación. La acreditación es el esfuerzo en conjunto de cada uno de los asistentes durante un periodo de 5 a 6 meses.

La esencia de los grupos de ayuda mutua radica en que los objetivos que se alcancen deben ser grupales, es decir, para considerarse un grupo "en control" deben cumplir en manera conjunta un nivel de hemoglobina glucosilada de $7 \%$ o menos de no ser así el grupo no se acredita y es esta acreditación la que marca el éxito del programa.

Los programas parten de la idea de que la educación comienza en casa y que los hábitos se heredan por lo cual para disminuir la prevalencia de la enfermedad en las próximas generaciones se crearon los programas y de esta forma los pacientes cuenten con una buena educación y sean auto eficaces.

Al conocer la estructura de cada uno de los programas, se decidió tomar el modelo educativo de DiabetIMSS para gamificar los temas de cada una de las sesiones dado que la secuencia de estos permite un aprendizaje secuencial, que parte desde los temas básicos hasta los especializados. Además, el director del hospital visitado dio acceso a la bibliografía y todos los materiales que los educadores utilizan para sus clases, los cuales contienen ejercicios propuestos, objetivos de cada unidad y la metodología para abordar cada uno de los temas.

Por recomendación de la Secretaría de Salud, se incorpora un módulo en el que antes de comenzar a utilizar la aplicación el paciente puede evaluar su nivel de riesgo a padecer diabetes a través de preguntas sobre su edad, peso y antecedentes familiares, esto con el objetivo de que el paciente conozca sus posibilidades de padecer diabetes y se interese en modificar sus hábitos alimenticios.

Con el material de trabajo de DiabetIMSS, se diseñó un plan de unidad basado en el planteamiento de Nérici [16] el cual indica que se debe tomar en cuenta el objetivo, materiales didácticos, la metodología de evaluación y las sub unidades que conformaran la unidad, adaptados a la tecnología a través de los elementos de gamificación que se seleccionaron a partir de la revisión de literatura y que eran los más utilizados en las aplicaciones:

- Puntos. Los puntos se otorgan por las actividades que se realizan en la aplicación como por ejemplo el registrar su peso, registrar una talla al día, realizar alguna actividad de algún módulo, entre otros.

- Medallas. Se proponen tres tipos de medallas: oro, plata y bronce, el color dependerá del porcentaje obtenido en la prueba final de la unidad. Las medallas tienen un nombre en específico de acuerdo a la unidad que se conteste, por ejemplo, se propone que si está en la unidad de alimentación, el nombre de la medalla sea "Comedor Saludable" (Fig. 1).

- Retroalimentación. Se proporcionará cada que el usuario conteste una pregunta dentro de las unidades de aprendizaje para que este obtenga información que complemente su respuesta o que le indique la respuesta correcta en caso de haber contestado de manera errónea y por qué esa era la opción correcta.

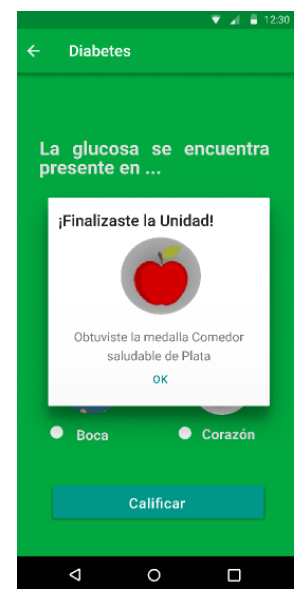

Figura 1. Ejemplo de medalla a otorgar
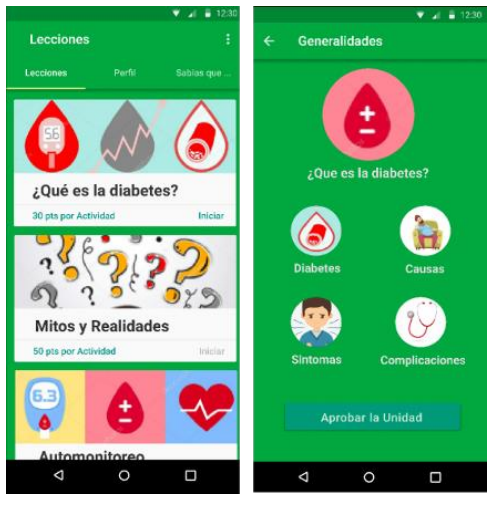

Figura 2. Módulo Lecciones
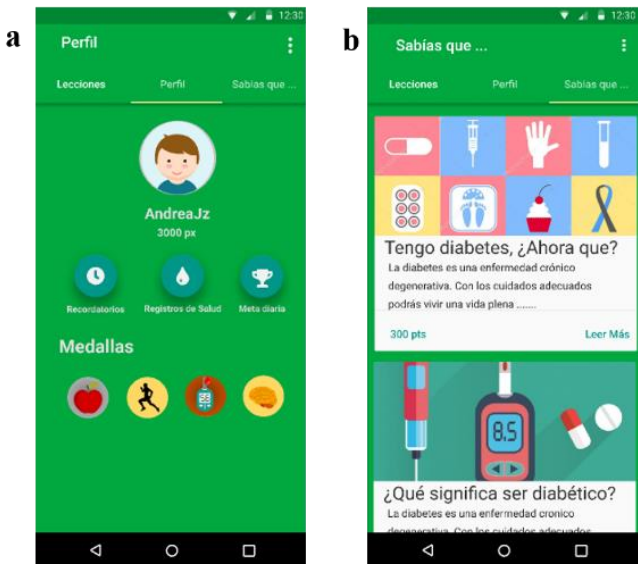

Figura 3. Pantallas de la aplicación. (a) Módulo Perfil. (b) Módulo ¿Sabías qué?

\section{Diseño de la aplicación}

La aplicación se diseñó para utilizarse en dispositivos móviles con sistema operativo Android ya que es un sistema disponible en la mayoría de los teléfonos de la población.

El contenido de la aplicación se organizó en cuatro módulos que cubren los temas de educación, actividad física y toma de medicamentos: 
a. Lecciones. Son los 12 temas del programa DiabetIMSS divididos en unidades (Figura 2). Cada unidad contiene ejercicios que le da puntos al usuario. Al término de todas las subunidades se activa un examen de evaluación de repaso de la unidad y cuando el paciente obtenga mínimo el $50 \%$ en la evaluación se le otorgará una medalla (dependiendo el porcentaje será el color de la medalla) con la que podrá acceder al contenido de la siguiente unidad.

b. Perfil. Presenta la información general del usuario y las medallas y puntos obtenidos hasta el momento (Figura 3a). Funciona como enlace a tres submódulos: 1) Recordatorios: el paciente puede generar alarmas que le recuerden la toma de medicamentos, realizar ejercicio, medir su nivel de azúcar en la sangre, entre otras, 2) Registros de salud, lleva el historial del peso, presión, azúcar y circunferencia abdominal y 3) Meta diaria, un reto donde el paciente define una meta diaria de pasos al día y que debe alcanzar para obtener una medalla.

c. ¿Sabías qué? Módulo informativo, presenta datos curiosos de la enfermedad en forma de tarjetas, así como consejos que le sirven al paciente para controlar su enfermedad (Figura 3b). Cada tarjeta aparece según el progreso en la aplicación y el tema de la unidad en la que se encuentre.

d. Evaluación de Riesgos. Aparece cuando se hace la primera visita a la aplicación. Inmediatamente después de registrarse dentro de la aplicación el sistema muestra preguntas en las cuales el paciente debe contestar si existen casos de pacientes diabéticos en su familia, la última vez que se tomó el nivel de azúcar, si toma medicamentos, su peso, talla, entre otros con la finalidad de indicarle si tiene riesgo incrementado de padecer diabetes o si está en los niveles prediabéticos.

\section{Evaluación}

Con el objetivo de detectar con anticipación posibles errores de usabilidad en el diseño del prototipo se realizó una prueba de evaluación heurística con 5 evaluadores expertos [17]. Se seleccionaron a evaluadores expertos en el área de la informática con experiencia en el diseño y desarrollo de interfaces.

Para la evaluación se creó una lista de áreas básicas como: 1) Contestar una unidad, 2) Registrar una meta, 3) Revisar el perfil, entre otras.

Los principios heurísticos utilizados fueron: 1) Visibilidad del estado del sistema, 2) Similitud entre el sistema y el mundo real, 3) Control y libertad del usuario, 4) Consistencia y cumplimiento de estándares, 5) Prevención de errores, 6) Preferencia al reconocimiento sobre la memorización, 7) Flexibilidad y eficiencia de uso, 8) Estética y diseño minimalista, 9) Ayuda ante errores, 10) Ayuda y documentación. Se midieron en una escala entre 0 (No es un problema) a 5 (Catástrofe).

Se pretende una segunda fase de esta evaluación en la que los evaluadores sean los usuarios finales con una versión completa de la aplicación para lograr la experiencia completa.

\section{Resultados}

Se tiene un prototipo funcional de la aplicación en el que se puede interactuar por cada uno de los módulos y con la información.

De la información recabada en las entrevistas se compararon los distintos programas de educación, se optó por DiabetIMSS y se tomó el material de trabajo de este. Con la evaluación se identificaron problemas de ayuda y documentación que se corregirán al inicio del desarrollo de la aplicación.

\section{Conclusiones}

La aplicación pretende servir como un recurso de apoyo en el que las personas con diabetes aprendan sobre su enfermedad acercándola a información confiable obtenida del IMSS y que los educadores utilizan en sus sesiones.

Con el uso de la gamificación se desea que las personas aprendan mientras obtienen reconocimientos por cada una de las actividades que van realizando y de esta manera comprometerlos a seguir utilizándola para así comprobar la eficacia de la gamificación.

\section{Referencias}

[1] American Diabetes Association, "2. Classification and Diagnosis of Diabetes", Diabetes Care, vol. 39, núm. Supplement 1, pp. S13-S22, ene. 2016.

[2] Observatorio Mexicano de Enfermedades No Transmisibles / Universidad Autónoma de Nuevo León, "Tablero de Control de Enfermedades Crónicas |OMENT". [En línea]. Disponible en: http://oment.uanl.mx/tablero-de-control-deenfermedades/. [Consultado: 07-sep-2017].

[3] R. A. DeFronzo, "Pathogenesis of type 2 diabetes mellitus", Med. Clin. North Am., vol. 88, núm. 4, pp. 787-835, jul. 2004.

[4] F. García et al., "Prevalencia de diabetes mellitus y factores de riesgo relacionados en una población urbana", Rev Soc Peru Med Interna, vol. 20, pp. 90-94, 2007.

[5] M. de los A. Vergara, "Formación de hábitos", Bol. Esc. Med., vol. 20, núm. 3, pp. 225-227, 1991.

[6] C. Duhigg, El poder de los hábitos. Books4pocket, 2015.

[7] I. Valadez, M. V. Farías, y N. A. Alfaro, "Educación para la Salud: la importancia del concepto", Rev. Educ. Desarro., vol. 1, núm. 6, pp. 43-48, 2004.

[8] G. Zichermann y C. Cunningham, Gamification by design: Implementing game mechanics in web and mobile apps. O’Reilly Media, Inc., 2011.

[9] A. F. Barbosa y F. G. Silva, "Serious Games: design and development of OxyBlood", en Proceedings of the 8th International Conference on Advances in Computer Entertainment Technology, 2011, p. 15.

[10] C. I. Muntean, "Raising engagement in e-learning through gamification", en Proc. 6th International Conference on Virtual Learning ICVL, 2011, pp. 323-329.

[11] "Nike+". [En línea]. Disponible en: https://www.nike.com/us/en_us/c/nike-plus/running-app-gps. [Consultado: 09-sep-2017].

[12] Duolingo, “Duolingo", Duolingo, 2017. [En línea]. Disponible en: https://es.duolingo.com/. [Consultado: 12-jun2017].

[13] [M. Glasemann, A. M. Kanstrup, y T. Ryberg, "Making chocolate-covered broccoli: designing a mobile learning game about food for young people with diabetes", 2010, p. 262.

[14] Masitoh, A. I. Wuryandari, y T. Mardiono, "Gamification on Steppy application in the development of the electronic health record as health service users of SHESOP", en Interactive Digital Media (ICIDM), 2015 4th International Conference on, 2015, pp. 1-6. 
[15] M. Takahashi, H. Kawasaki, A. Maeda, y M. Nakamura, "Mobile walking game and group-walking program to enhance going out for older adults", 2016, pp. 1372-1380.

[16] I. G. Nérici, Hacia una didactica general dinamica. Buenos Aires: Kapeluz, 1996.
[17] J. Nielsen, "How to conduct a heuristic evaluation", Nielsen Norman Group, vol. 1, pp. 1-8, 1995. 